OPEN ACCESS

Edited by:

Xiancang $\mathrm{Ma}$,

First Affiliated Hospital of Xi'an

Jiaotong University, China

Reviewed by:

Edita Fino,

University of Bologna, Italy

Francesco Marchini,

Consultant, Rome, Italy

${ }^{*}$ Correspondence:

Chengping Hu

chengpinghu2021@163.com

Luo Qiong

lq1499964084@163.com

tThese authors have contributed equally to this work and share first

authorship

Specialty section:

This article was submitted to

Psychopathology,

a section of the journal

Frontiers in Psychology

Received: 01 June 2021 Accepted: 28 September 2021 Published: 26 October 2021

Citation:

Wang L, Zhang Y, Zhang X, Fan X, Qiong L and Hu C (2021) The Potential Impact of Childhood

Traumatic Experiences on Coping

Styles and Emotion Regulation

of Nurse Practitioners During

the COVID-19 Outbreak.

Front. Psychol. 12:718780

doi: 10.3389/fpsyg.2021.718780

\section{The Potential Impact of Childhood Traumatic Experiences on Coping Styles and Emotion Regulation of Nurse Practitioners During the COVID-19 Outbreak}

\author{
Liyuanke Wang ${ }^{1 t}$, Yan Zhang ${ }^{1 t}$, Xiaoli Zhang ${ }^{1 t}$, Xiwang Fan ${ }^{2 t}$, Luo Qiong ${ }^{1 *}$ and \\ Chengping $\mathrm{Hu}^{2 *}$
}

${ }^{1}$ Suining Central Hospital, An Affiliated Hospital of Chongqing Medical University, Suining, China, ${ }^{2}$ Department of Psychiatry, Shanghai Pudong New Area Mental Health Center, Tongji University School of Medicine, Shanghai, China

Background: During an epidemic of a novel infectious disease, frontline medical staff suffer from high psychological stress. Previous studies have found that traumatic childhood experiences are associated with mental and physical health in adulthood. Anxiety and depression were measured and analyzed in relation to childhood trauma and coping styles. This study aims to explore the correlational study between traumatic childhood experiences and coping styles among nurse practitioners.

Method: This study sampled 278 nurse practitioners from hospitals designated for the treatment of the novel coronavirus in Sichuan Province. The study measures included the Simplified Coping Style Questionnaire and the Childhood Trauma QuestionnaireShort Form. This research intends to use correlational study methods to explore the relationship between the two factors.

Results: Statistical analysis showed that there was no statistically significant difference in the general demographic data between the two groups.

Conclusion: Childhood traumatic experiences have a significant impact on the active coping of nurse practitioners, and active coping may be emotionally protective for nurse practitioners.

Keywords: childhood traumatic experiences, coping styles, nurse practitioners, correlation, COVID-19

\section{INTRODUCTION}

COVID-19 first occurred in late 2019 and has rapidly spread to most countries and regions around the world (Bchetnia et al., 2020). The sudden increase in diagnosed cases puts tremendous pressure on frontline medical staff, and nurse practitioners, as important clinical workers, work in a high-stress and high-intensity environment, which poses threats to their psychological wellbeing (Falcone et al., 2020; Xu et al., 2020). Front-line clinical staff mental health can have an impact on the physician-patient relationship (Marchini et al., 2021). Previous studies have found that traumatic childhood experiences are associated with mental and physical health in adulthood 
TABLE 1 | General characteristics of the study participants.

\begin{tabular}{lcccc}
\hline Characteristics & All & \multicolumn{1}{c}{ Non-trauma group Trauma group $\boldsymbol{p}$-value } \\
\hline Gender (male/female) & $10 / 268$ & $7 / 154$ & $3 / 114$ & 0.432 \\
Age (years) & 40.69 & $19.56 \pm 2.012$ & $19.62 \pm 2.188$ & 0.824 \\
Grade & & & & 0.074 \\
Technical secondary school & 31 & 24 & 7 & \\
Junior college & 242 & 133 & 109 & \\
University education & 5 & 4 & 1 & \\
\hline
\end{tabular}

Data are presented as the mean $\pm S D$.

(Felitti et al., 1998; Anda et al., 2009). Additionally, coping styles have an impact on mental health (Ma et al., 2018). Therefore, this study aims to explore the correlation between traumatic childhood experiences and coping styles among nurse practitioners.

\section{METHODS}

This study sampled 278 nurse practitioners from hospitals designated for the treatment of the novel coronavirus in Sichuan Province. The study measures included the Simplified Coping Style Questionnaire (SCSQ), Self-Rating Depression Scale (SDS), Self-Rating Anxiety Scale (SAS), and Childhood Trauma Questionnaire-Short Form (CTQ-SF). CTQ-SF and a tool developed by the American clinical psychologist Bernstein to measure childhood abuse (Bernstein et al., 1997). There are 25 clinical entries and 3 validity entries for a total of 28 entries (Bernstein et al., 2003). The revised questionnaire introduced by Chinese researchers had five dimensions: emotional abuse, physical abuse, sexual abuse, physical neglect, and emotional neglect ( $\mathrm{Li}$ et al., 2014). It has been shown that the Chinese version of the childhood trauma questionnaire had good reliability and validity in China (Xie et al., 2018). The study was approved by the ethical review committee of Suining Central Hospital. All nurse practitioners specify informed consent procedures. Statistical analysis was performed using the SPSS 21.0 software package (SPSS, Chicago, IL, United States).

\section{RESULTS}

Descriptive and statistical comparisons of the general characteristics of the study participants are shown in Table 1.

Statistical analysis showed that there was no statistically significant difference in the general demographic data between the two groups.

Significant differences in SAS, SDS and active coping scores were observed between the two groups $(p<0.001)$ in Table 2 . However, the difference in passive coping style scores between the two groups was not statistically significant (Table 2).

There was a significant negative correlation between positive coping style and anxiety and depression in the trauma group, with correlation coefficients ranging from 0.3 to 0.5 (Table 3). It is
TABLE 2 | Comparative analysis of coping styles and emotional scores in childhood trauma and non-trauma groups.

\begin{tabular}{lcccc}
\hline & Trauma group & Non-trauma group & $\boldsymbol{t}$ & $\boldsymbol{p}$ \\
\hline SAS & $41.92 \pm 10.97$ & $37.09 \pm 6.02$ & -4.69 & 0.000 \\
SDS & $55.98 \pm 6.19$ & $52.56 \pm 4.67$ & -5.24 & 0.000 \\
Active coping & $21.15 \pm 7.30$ & $25.02 \pm 6.12$ & 4.80 & 0.000 \\
Passive coping & $8.60 \pm 4.26$ & $8.83 \pm 3.76$ & 0.48 & 0.636 \\
\hline
\end{tabular}

SDS, Self-Rating Depression Scale; SAS, Self-Rating Anxiety Scale.

TABLE 3 | Correlation of coping styles among the trauma group with anxiety and depression.

\begin{tabular}{lcc} 
& Active coping & Passive coping \\
\hline SAS & $-0.36^{\star \star}$ & $0.18^{\star}$ \\
SDS & $-0.52^{\star \star}$ & 0.09
\end{tabular}

${ }^{* *}$ Correlation is significant at a confidence level of 0.01. ${ }^{*}$ Correlation is significant at a confidence level of 0.05 .

assumed that a positive coping style has a protective effect against anxiety and depression.

\section{DISCUSSION}

Childhood trauma experiences are negatively correlated with individual coping styles, and individuals with childhood trauma are less likely to develop positive psychological cues and positive coping styles (Edinger et al., 2020; Wan et al., 2020). Childhood trauma experiences can be biologically embedded in the brain, leading to long-term activation of the stress response system as well as anxiety and depression (Bellis et al., 2017; Bryan and Beitz, 2019). Coping styles refer to the way an individual perceives and behaves in response to an environment beyond his or her control due to internal and external factors (Flewelling et al., 2020; Rohleder et al., 2020). Passive coping styles, such as avoidance and fantasy, are associated with psychological symptoms such as anxiety and depression; individuals who adopt positive coping styles have stable mechanisms for regulating their emotions and are more likely to feel positive emotions (Jones et al., 2016; Gao et al., 2021). At the same time, previous studies have found that the protective effect of coping style on effects of war trauma exposure (Fino et al., 2020). The importance of emotion regulation skills especially in healthcare professionals like nurses who are routinely exposed to high stress and emotionally salient situations (Fino et al., 2021). The relevance of childhood traumatic experiences in terms of implications for psychosocial support programs in healthcare personnel (Fino et al., 2019).

\section{CONCLUSION}

In summary, childhood traumatic experiences have a significant impact on the active coping of nurse practitioners, and active coping may be emotionally protective for nurse practitioners. The limits of the present study and state the need to verify these results in a more robust design of research. 


\section{DATA AVAILABILITY STATEMENT}

The original contributions presented in the study are included in the article/supplementary material, further inquiries can be directed to the corresponding author.

\section{AUTHOR CONTRIBUTIONS}

$\mathrm{CH}$ and LQ contributed to the conception and design of the present study. LW recruited the patients and conducted the study. YZ, XZ, and XF undertook the statistical analyses and wrote the first manuscript draft. All authors contributed to the data analysis and drafting and revision of the article, gave final approval of the version to be

\section{REFERENCES}

Anda, R. F., Dong, M., Brown, D. W., Felitti, V. J., Giles, W. H., Perry, G. S., et al. (2009). The relationship of adverse childhood experiences to a history of premature death of family members. BMC Public Health 9:106. doi: 10.1186/ 1471-2458-9-106

Bchetnia, M., Girard, C., Duchaine, C., and Laprise, C. (2020). The outbreak of the novel severe acute respiratory syndrome coronavirus 2 (SARS-CoV-2): A review of the current global status. J. Infect. Public Health 13, 1601-1610. doi: 10.1016/j.jiph.2020. 07.011

Bellis, M. A., Hardcastle, K., Ford, K., Hughes, K., Ashton, K., Quigg, Z., et al. (2017). Does continuous trusted adult support in childhood impart life-course resilience against adverse childhood experiences - a retrospective study on adult health-harming behaviours and mental well-being. BMC Psychiatry 17:110. doi: 10.1186/s12888-017-1 $260-\mathrm{z}$

Bernstein, D. P., Ahluvalia, T., Pogge, D., and Handelsman, L. (1997). Validity of the Childhood Trauma Questionnaire in an adolescent psychiatric population. J. Am. Acad. Child Adolesc. Psychiatry 36, 340-348. doi: 10.1097/00004583199703000-00012

Bernstein, D. P., Stein, J. A., Newcomb, M. D., Walker, E., Pogge, D., Ahluvalia, T., et al. (2003). Development and validation of a brief screening version of the Childhood Trauma Questionnaire. Child Abuse. Negl. 27, 169-190. doi: 10.1016/s0145-2134(02)00541-0

Bryan, R., and Beitz, J. M. (2019). Connections among biologic embedding of childhood adversity, adult chronic illness, and wound care: a review of the literature. Wound. Manag. Prev. 65, 18-28. doi: 10.25270/wmp.2019.10. 1828

Edinger, A., Fischer-Waldschmidt, G., Parzer, P., Brunner, R., Resch, F., and Kaess, M. (2020). The impact of adverse childhood experiences on therapy outcome in adolescents engaging in nonsuicidal self-injury. Front. Psychiatry 11:505661. doi: $10.3389 /$ fpsyt.2020.505661

Falcone, R., Grani, G., Ramundo, V., Melcarne, R., Giacomelli, L., Filetti, S., et al. (2020). Cancer Care During COVID-19 Era: the quality of life of patients with thyroid malignancies. Front. Oncol. 10:1128. doi: 10.3389/fonc.2020. 01128

Felitti, V. J., Anda, R. F., Nordenberg, D., Williamson, D. F., Spitz, A. M., Edwards, V., et al. (1998). Relationship of childhood abuse and household dysfunction to many of the leading causes of death in adults. The Adverse Childhood Experiences (ACE) Study. Am. J. Prev. Med. 14, 245-258. doi: 10.1016/s07493797(98)00017-8

Fino, E., Bonfrate, I., Fino, V., Bocus, P., Russo, P. M., and Mazzetti, M. (2021). Harnessing distress to boost growth in frontline healthcare workers during COVID-19 pandemic: the protective role of resilience, emotion regulation and social support. Psychol. Med. 2021, 1-3. doi: 10.1017/s0033291721000519 published, and agreed to be accountable for all aspects of the work.

\section{FUNDING}

This study was supported by the Shanghai Pudong Municipal Health Commission (PWRI2020-11) and Assessment of the Psychological Status of COVID-19 Infected Population and Associated Clinical Staff and Study of the Effectiveness of Psychological Crisis Intervention (2020PTJSGG009).

\section{ACKNOWLEDGMENTS}

We thank all patients who volunteered to participate in the study.

Fino, E., Di Campli, S., Patrignani, G., and Mazzetti, M. (2019). The modulating role of gender and aggression in emotional reactions of nursing students: A cross-sectional study. J. Adv. Nurs. 75, 1462-1472. doi: 10.1111/jan. 13936

Fino, E., Mema, D., and Russo, P. M. (2020). War trauma exposed refugees and posttraumatic stress disorder: The moderating role of trait resilience. J. Psychosom. Res. 129:109905. doi: 10.1016/j.jpsychores.2019.10 9905

Flewelling, K. D., Koehler, A., Shaffer, J., and Dill, E. J. (2020). Correlates of health-related quality of life in youth with psychogenic nonepileptic seizures. Seizure 83, 203-207. doi: 10.1016/j.seizure.2020. 09.030

Gao, T., Mei, S., Li, M., Arcy, C., and Meng, X. (2021). Roles of Psychological Distress and Social Support in the Relationship Between Childhood Maltreatment and Perceived Needs for Mental Health Care. J. Interpers Viol. 2021:8862605211006368.

Jones, B., Reuber, M., and Norman, P. (2016). Correlates of health-related quality of life in adults with psychogenic nonepileptic seizures: a systematic review. Epilepsia 57, 171-181. doi: 10.1111/epi.13268

Li, X. B., Liu, J. T., Zhu, X. Z., Zhang, L., Tang, Y. L., and Wang, C. Y. (2014). Childhood trauma associates with clinical features of bipolar disorder in a sample of Chinese patients. J. Affect. Disord. 168, 58-63. doi: 10.1016/j.jad.2014. 06.017

Ma, S., Wan, Y., Zhang, S., Xu, S., Liu, W., Xu, L., et al. (2018). Mediating effect of psychological symptoms, coping styles and impulsiveness on the relationship between childhood abuses and non-suicidal self-injuries among middle school students. Wei Sheng Yan Jiu 47, 530-535.

Marchini, F., Caputo, A., Convertino, A., and Napoli, A. (2021). Psychodynamics in diabetes: the relevance of deepening the symbolic in treatment adherence. Front. Psychol. 12:661211. doi: 10.3389/fpsyg.2021.66 1211

Rohleder, C., Song, Y. J. C., Crouse, J. J., Davenport, T. A., Iorfino, F., Hamilton, B., et al. (2020). Youth Mental Health Tracker: protocol to establish a longitudinal cohort and research database for young people attending Australian mental health services. BMJ Open 10:e35379. doi: 10.1136/bmjopen-2019-03 5379

Wan, Y., Chen, R., Wang, S., Clifford, A., Zhang, S., Orton, S., et al. (2020). Associations of coping styles with nonsuicidal self-injury in adolescents: Do they vary with gender and adverse childhood experiences? Child Abuse. Negl. 104:104470. doi: 10.1016/j.chiabu.2020.10 4470

Xie, P., Wu, K., Zheng, Y., Guo, Y., Yang, Y., He, J., et al. (2018). Prevalence of childhood trauma and correlations between childhood trauma, suicidal ideation, and social support in patients with depression, bipolar disorder, and schizophrenia in southern China. J. Affect. Disord. 228, 41-48. doi: 10.1016/j. jad.2017.11.011 
Xu, J., Xu, Q. H., Wang, C. M., and Wang, J. (2020). Psychological status of surgical staff during the COVID-19 outbreak. Psychiatry Res. 288:112955. doi: 10.1016/j.psychres.2020.112955

Conflict of Interest: The authors declare that the research was conducted in the absence of any commercial or financial relationships that could be construed as a potential conflict of interest.

Publisher's Note: All claims expressed in this article are solely those of the authors and do not necessarily represent those of their affiliated organizations, or those of the publisher, the editors and the reviewers. Any product that may be evaluated in this article, or claim that may be made by its manufacturer, is not guaranteed or endorsed by the publisher.

Copyright () 2021 Wang, Zhang, Zhang, Fan, Qiong and Hu. This is an open-access article distributed under the terms of the Creative Commons Attribution License (CC BY). The use, distribution or reproduction in other forums is permitted, provided the original author(s) and the copyright owner(s) are credited and that the original publication in this journal is cited, in accordance with accepted academic practice. No use, distribution or reproduction is permitted which does not comply with these terms. 